\title{
Desmoid Tumor Causing Hip Joint Contracture: A Case Report
}

\author{
Kazuhiko Hashimoto*, Shunji Nishimura, Kensuke Toriumi, Shunki Iemura, Naohiro Oka, \\ Masao Akagi
}

Department of Orthopedic Surgery, Kindai University Hospital, Osaka-Sayama City, Japan

Email: *hazzhiko@med.kindai.ac.jp

How to cite this paper: Hashimoto, K., Nishimura, S., Toriumi, K., Iemura, S., Oka, N. and Akagi, M. (2018) Desmoid Tumor Causing Hip Joint Contracture: A Case Report. Open Journal of Orthopedics, 8, 175-182.

https://doi.org/10.4236/ojo.2018.85020

Received: April 7, 2018

Accepted: May 8, 2018

Published: May 11, 2018

Copyright $\odot 2018$ by authors and Scientific Research Publishing Inc. This work is licensed under the Creative Commons Attribution International License (CC BY 4.0).

http://creativecommons.org/licenses/by/4.0/

\begin{abstract}
Desmoid tumor (DT) is a relatively uncommon, cytologically bland fibrous neoplasm that is associated with possibility of local recurrence but without the potential to spread to other parts of the body. This disease affects mostly younger adults and is the most common cause of abdominal wall masses in women 20 - 35 years of age. However, it may involve nearly every body part, including the extremities, head and neck, trunk, and abdominal cavity; as such, patients with DT may present to a range of general and subspecialty radiologists. The consensus for treatment has changed over the past decade, with most centers moving away from primary radical surgery towards a front-line "wait-and-see" policy. Here, we present a case of a tumor mass that induced hip joint contracture. DT does not usually cause functional disorder. Oncologists should be aware that a desmoid tumor can cause hip joint contracture if the mass occurs in the gluteal region. Moreover, DT resulting in such a functional disorder should be treated by resection.
\end{abstract}

\section{Keywords}

Desmoid Tumor, Hip Joint Contracture, Gluteal Region

\section{Introduction}

Desmoid tumor (DT) is defined as a borderline lesion of the connective tissue with a tendency to infiltrate adjacent tissue, but without metastatic potential, according to the World Health Organization's classification [1]. DT is characterized by local aggressiveness with a $20 \%$ - 30\% local recurrence rate, but without metastasis [1] [2]. In a previous study, the median age of patients was reported to be 12.2 years; $49 \%$ of patients were male, and $51 \%$ were female [3]. DT most commonly arises in the extremities (40\%), thorax (23\%), head and neck (21\%), 
and trunk (16\%) [3]. Diagnosis is generally confirmed with histological findings of spindle cells and fibroblasts infiltrating the adjacent tissue in fascicles. Mitoses may be infrequent. The European Organisation for Research and Treatment of Cancer (EORTC)/Soft Tissue and Bone Sarcoma Group has suggested that a "watch and wait strategy" is the first choice in the treatment of DT, while wide resection should be considered as a treatment option if postoperative morbidity is acceptable [4]. The incidence of pain in patients with DT is approximately $63 \%$ [5]. However, functional disorder by DT has not previously been reported. Herein, we describe the first known case of DT to cause hip joint contracture.

\section{Case Presentation}

A 37-year-old woman visited our hospital with pain in the left gluteal region and difficulty in external rotation of her left leg for the previous 3 years. She had no related traumatic or medical history. She did, however, have a history of two pregnancies and births. The patient showed a hump on her gluteal region. The mass was $12 \times 8 \mathrm{~cm}$ in size. The patient had spontaneous pain in her gluteal region, and there was also tenderness on the mass. There was no numbness in her leg. The active range of motion of her hip joint was an internal rotation of $45^{\circ}$ and external rotation of $0^{\circ}$.

Computed tomography (CT) images showed a mass on her gluteal region (Figure 1(a) \& Figure 1(b)). Tumorous lesions on the left buttocks appeared low on T1- and T2-weighted images, while the signal was partly high in magnetic resonance imaging (MRI), which revealed subcutaneous invasion. An enhancement effect was observed by gadolinium-enhanced MRI (Figure 2). No accumulation was observed on thallium-201 scintigraphy (Figure 3). We conducted a needle biopsy guided by CT scanning. Histology revealed an increase in connective tissue. Spindle-like cells were bundled and growing in spiral patterns

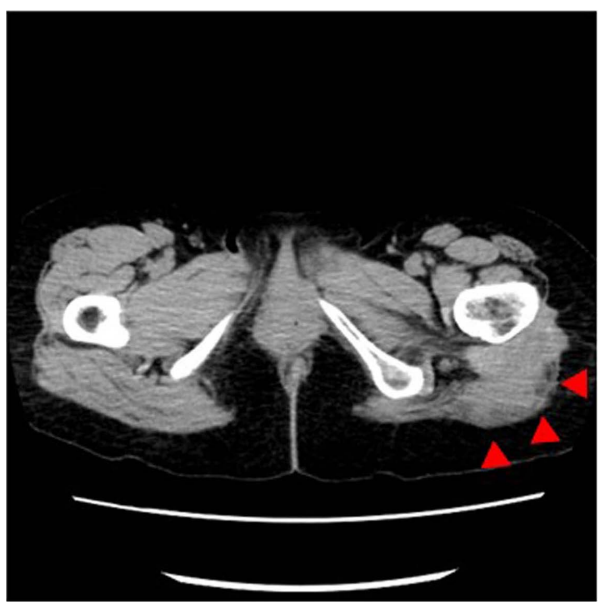

(a)

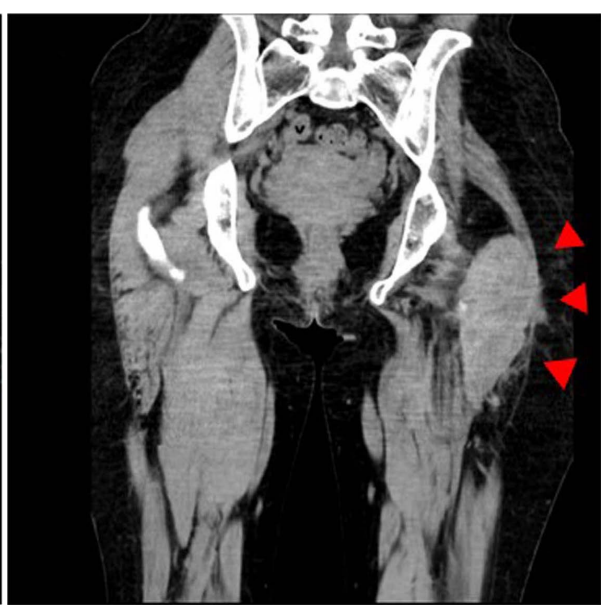

(b)

Figure 1. Computed tomography (CT) images of the tumor. (a) CT scan showing a sagittal image of the tumor mass in the left gluteal region; (b) CT scan showing a coronal image of the tumor mass in the left gluteal region. Arrowheads indicate the tumor mass. 


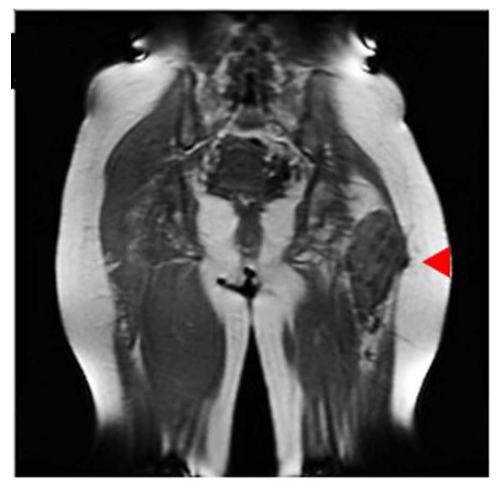

(a)

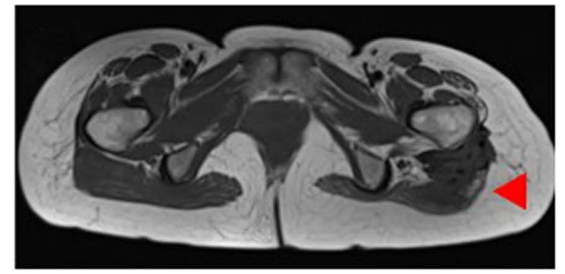

(b)

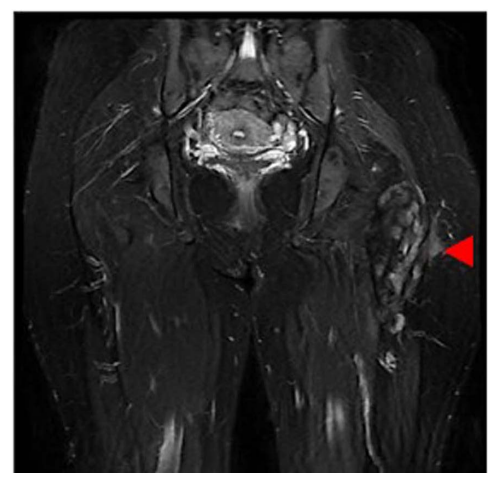

(c)

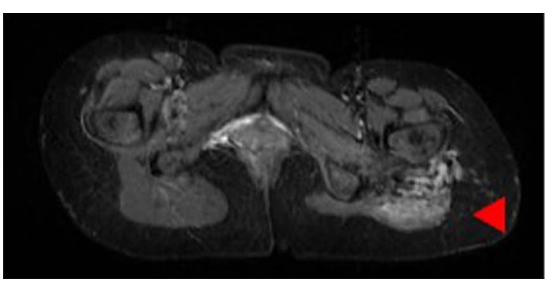

(d)

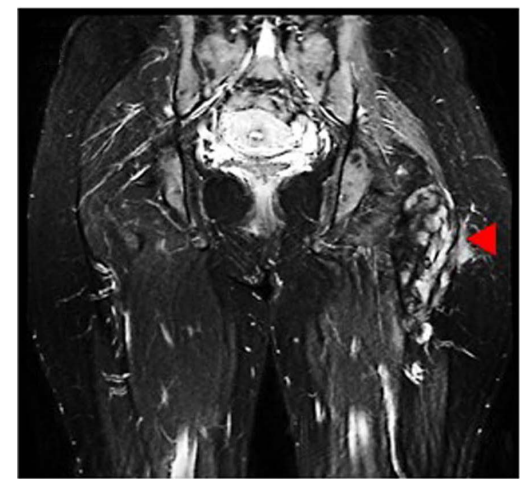

(e) 


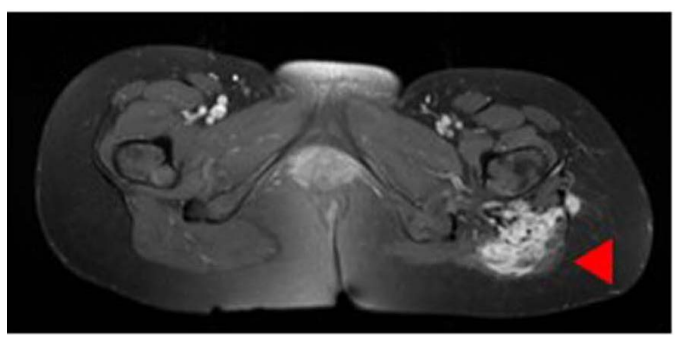

(f)

Figure 2. Magnetic resonance images of the tumor mass in the left gluteal region. (a) T1-weighted coronal image; (b) T1-weighted sagittal image; (c) T2-weighted coronal image; (d) T2-weighted sagittal image; (e) Contrast-enhanced coronal image; (f) Contrast-enhanced sagittal image. Arrowheads indicate the tumor mass.

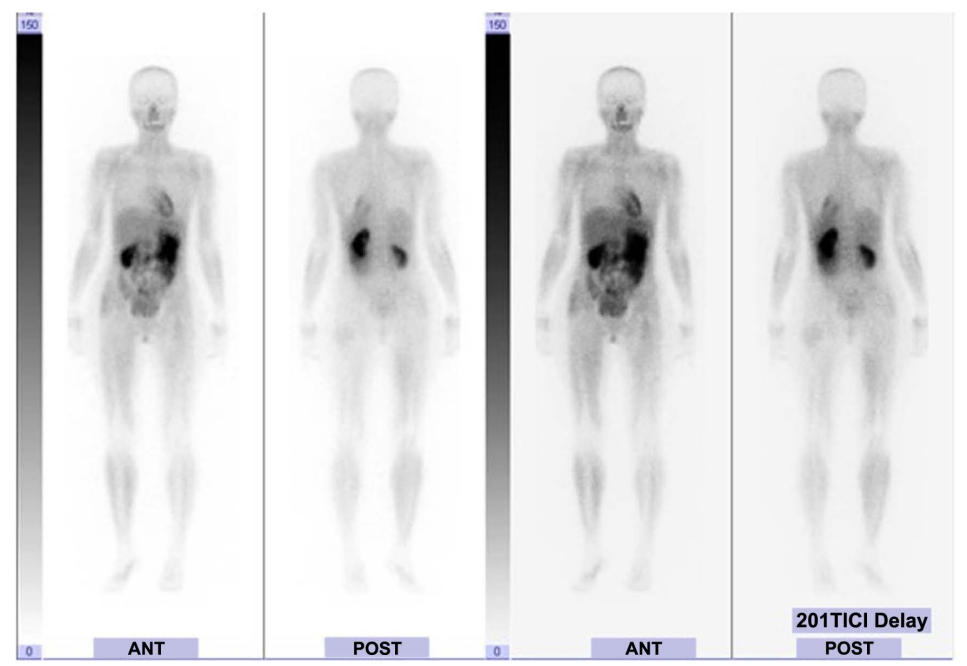

Figure 3. Thallium-201 scintigraphy image of the whole body. No accumulation was observed in the tumor region.

(data not shown). No heterozygosity was observed in the nuclei of the growing cells.

We suspected DT, and subsequently performed extensive resection. The tumor had adhered to the fascia of the short rotator muscle group, but because there was no invasion into the muscle, the fascia was placed on the tumor side and resected, leaving the short rotator muscle group (Figure 4). Hip contracture was relieved immediately after surgery. Macroscopically, the resected specimen was margin-free (Figure 4). The histology of the resected specimen showed growing spindle cells with no nuclear dysplasia (Figure 5). We did not administer adjuvant therapy because the histological margin was negative. In the 3 years after surgery, we have not observed recurrence, metastasis, or hip joint contracture.

\section{Discussion}

DT accounts for about 3\% of soft tissue tumors, about $15 \%$ of which are extra-peritoneal DT [6]. The majority of patients present with painless swelling. 


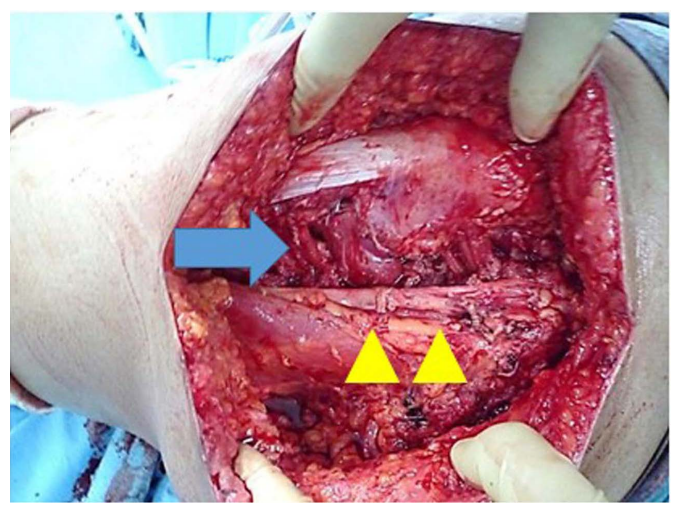

(a)

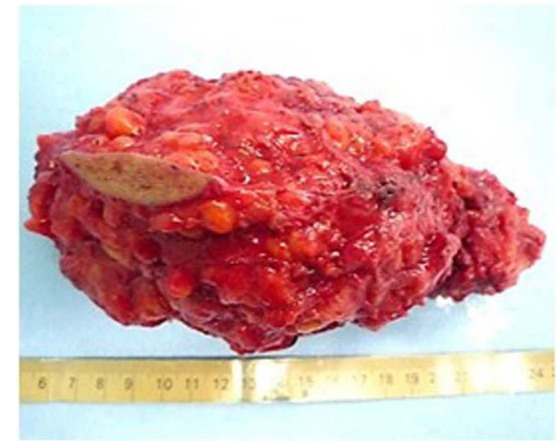

(b)

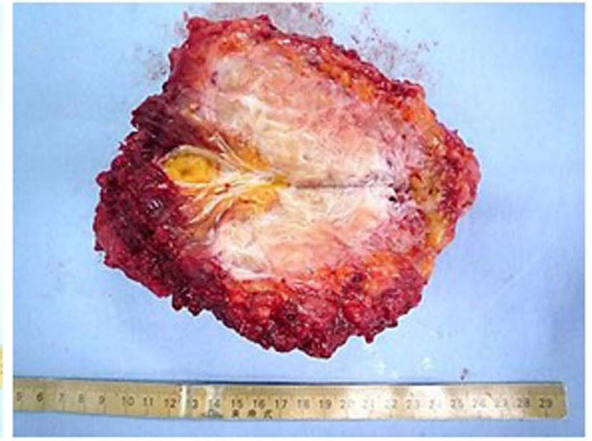

(c)

Figure 4. Surgically resected tissues. (a) Surgical site findings after the tumor was resected; (b) macroscopic image of the resected specimen; (c) the cut surface of the resected specimen. The macroscopic margin was clear, and the tumor was elastic and hard. The cut surface was white. The blue arrow indicates the short rotator muscle, while yellow arrowheads show the sciatic nerve.

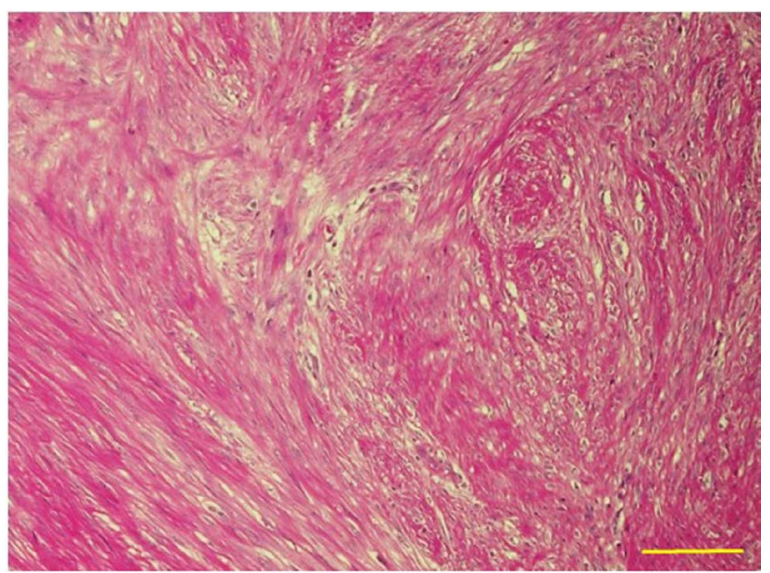

Figure 5. Hematoxylin and eosin staining of the resected specimen. Scale bar $=100 \mu \mathrm{m}$.

However, should a lesion have adhered to deep structures, patients may present with contractures, pain, and dysfunction. When lesions expand and compress or invade local nerves, neuropathy may subsequently develop, resulting in in radiculopathy, paresthesia, or even motor weakness [6]. However, no cases of DT have been reported in which the tumor caused hip joint contracture-the 
present report describes the first such case. In our patient, we hypothesize that because the tumor was large and adhered to the short rotator muscle group, the hip joint contracture occurred as a result of the pressure.

$\mathrm{CT}$ is of limited value given the similar attenuation between muscle and extra-abdominal desmoid lesions, as illustrated in the current study [6]. MRI is the modality of choice to assess both the size of the mass and its intimate association with surrounding structures. Lesions on T1-weighted images are homogenously isointense with a high heterogenous signal on T2-weighted images [7]. Although no accumulation was detected in the present DT case by thallium-201 scintigraphy, a previous report showed that DT could be detected by this methodology [8]. The current DT may have had lower activity than that of the previous case [8].

In histological findings, DT usually shows spindle cells in fascicles, with infiltration into muscles [9]. However, DTs do not have metastatic potential and therefore can be treated with a "wait-and-see" policy. In a retrospective analysis, Fiore et al. demonstrated that $50 \%$ of patients benefited from a nonaggressive front-line policy [10]. However, cases of DT that cause functional disorders, such as the present case, cannot be treated with such a wait-and-see strategy.

Wide-margin surgical resection, despite its high rate of recurrence, has traditionally been the first-line treatment option [11]. Huang et al. recently reported a univariate analysis showing that admission status (primary/recurrent), gender, tumor size, and margin status are all correlated with local recurrence, while size and margin status were independent prognostic factors in multivariate analysis. The local recurrence-free survival rate for primary disease at 5 years was $64 \%$ for those with positive margins, compared to $92 \%$ at the same time point for those with negative margins [12]. We utilized a wide margin because we suspected that margin status would be important for obtaining a good prognosis in the current case.

Low grade lesions with no metastatic potential, such as DT, should in theory not respond to chemotherapeutic agents given their low cell turnover rates. With an increased knowledge of these lesions at the cellular level, responses to chemotherapy have been demonstrated, thus defying the traditional belief held at an oncological level [6].

The use of postoperative radiation, as described in a review of 22 case series by Nuyttens et al. [13], resulted in an increase in the local control of the disease. In patients with positive margins, local control was increased from $4 \%$ to $75 \%$ with the addition of adjuvant irradiation. There was also a positive result when irradiation was used in patients with negative surgical margins. In the current study, we did not administer radiation treatment because the margin was negative.

\section{Conclusion}

DT is a benign tumor, but it sometimes causes functional disorders such as hip joint contracture. If an oncologist observes DT in the gluteal region, they should 
consider that the DT may cause hip joint contracture. Moreover, the tumor should be resected before any functional disorder occurs.

\section{Acknowledgements}

We would like to thank Editage (https://www.editage.jp/) for English language editing.

\section{Informed Consent}

We obtained agreement from the patient to publish this case report.

\section{References}

[1] Benej, R., Mečiarová, I. and Pohlodek, K. (2017) Desmoid-Type Fibromatosis of the Breast: A Report of 2 Cases. Oncology Letters, 14, 1433-1438. https://doi.org/10.3892/ol.2017.6337

[2] Barnes, L., Eveson,J.W., Reichart, P. and Sidransky, D. (2005) World Health Organization Classification of Tumors. Pathology and Genetics of Head and Neck Tumors. IARC Press, Lyon.

[3] Ver Halen, J.P., Soto-Miranda, M.A. and Sandoval, J.A. (2015) Reconstruction of Desmoid Tumors: Case Series and Systematic Review. Annals of Plastic Surgery, 75, 480-486. https://doi.org/10.1097/SAP.0000000000000129

[4] Kasper, B., Baumgarten, C., Bonvalot, S., Haas, R., Haller, F., Hohenberger, P., et al. (2015) Management of Sporadic Desmoid-Type Fibromatosis: A European Consensus Approach Based on Patients' and Professionals' Expertise-A Sarcoma Patients EuroNet and European Organisation for Research and Treatment of Cancer/Soft Tissue and Bone Sarcoma Group Initiative. European Journal of Cancer, 51, 127-136. https://doi.org/10.1016/j.ejca.2014.11.005

[5] Rigaux, P., Lefebvre-Kuntz, D. and Penel, N., "SOS Desmoïde” (2015) Pain Burden in Desmoid Tumor Patients: A Survey of the French Advocacy Group SOS Desmoid. Bulletin du Cancer, 102, 213-216. https://doi.org/10.1016/j.bulcan.2015.02.001

[6] Molloy, A.P., Hutchinson, B. and O’Toole, G.C. (2012) Extra-Abdominal Desmoid Tumours: A Review of the Literature. Sarcoma, 2012, 578052. https://doi.org/10.1155/2012/578052

[7] O'Keefe, F., Kim, E.E. and Wallace, S. (1990) Magnetic Resonance Imaging in Aggressive Fibromatosis. Clinical Radiology, 42, 170-173. https://doi.org/10.1016/S0009-9260(05)81927-0

[8] Murata, H., Kusuzaki, K., Hirata, M., Hashiguchi, S. and Hirasawa, Y. (2000) Extra abdominal Desmoid Tumor with Dissemination Detected by Thallium-201 Scintigraphy. Anticancer Research, 20, 3963-3966.

[9] Weiss, S.W. and Goldblum, J.R. (2001) Fibromatoses. In: Eizinger and Weiss's Soft Tissue Tumors, Mosby, Maryland Heights, MO, 309-346.

[10] Fiore, M., Rimareix, F., Mariani, L., Domont, J., Collini, P., Le Péchoux, C., et al. (2009) Desmoid-Type Fibromatosis: A Front-Line Conservative Approach to Select Patients for Surgical Treatment. Annals of Surgical Oncology, 16, 2587-2593. https://doi.org/10.1245/s10434-009-0586-2

[11] Dalén, B.P., Bergh, P.M. and Gunterberg, B.U. (2003) Desmoid Tumors: A Clinical Review of 30 Patients with More than 20 Years' Follow-Up. Acta Orthopaedica Scandinavica, 74, 455-459. https://doi.org/10.1080/00016470310017785 
[12] Huang, K., Fu, H., Shi, Y.Q., Zhou, Y. and Du, C.Y. (2009) Prognostic Factors for Extra-Abdominal and Abdominal Wall Desmoids: A 20-Year Experience at a Single Institution. Journal of Surgical Oncology, 100, 563-569.

https://doi.org/10.1002/jso.21384

[13] Nuyttens, J.J., Rust, P.F., Thomas Jr., C.R. and Turrisi 3rd., A.T. (2000) Surgery versus Radiation Therapy for Patients with Aggressive Fibromatosis or Desmoid Tumors: A Comparative Review of 22 Articles. Cancer, 88, 1517-1523.

https://doi.org/10.1002/(SICI)1097-0142(20000401)88:7<1517::AID-CNCR3>3.0.C $\underline{\mathrm{O} ; 2-9}$ 